\title{
LA PESQUERÍA DEL LANGOSTINO ARGENTINO PLEOTICUS MUELLERI (CRUSTACEA: PENAEIDAE) EN PATAGONIA, ¿UN ÚNICO STOCK?
}

\author{
ARGENTINE SHRIMP FISHERY PLEOTICUS MUELLERI (CRUSTACEA: \\ PENAEIDAE) IN PATAGONIA, IS IT A SINGLE STOCK?
}

Pedro De Carli ${ }^{1,2,}$, Juan C. Braccalenti ${ }^{1,2}$, Francisco J. García-De-León ${ }^{3,4}$ \& Eliana P. Acuña Gómez ${ }^{3}$

\begin{abstract}
Argentine shrimp fishery in Patagonia was managed since its beginning as a single stock. The fishery research done on this issue considered that the concept of stock unity and cohort monitoring could not be applied to Argentine shrimp fishery. The annual landings of shrimp presented significant variations in number, which could not been explained in an effective manner. This paper analyses the feasibility of recognizing different subunits of stock. The biological information available enables the recognition of three

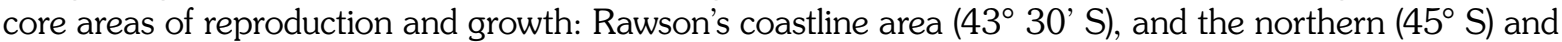
southern $\left(47^{\circ} \mathrm{S}\right)$ areas of the San Jorge Gulf. The available results of mark-recapture experiments indicate that a fraction of the population is born and develops in the southern area, remains in this area and will probably make a breeding contribution to the next generation. In the last decade, in the year 2001 when annual catches were the highest, the concentration of fishing activity in Rawson's coastal area was found to be characterized by maximum yields and sizes. Nevertheless, these evidences are not sufficient and require other methods in order to differentiate the three proposed stock subunits. A population genetic analysis using molecular markers is proposed as a robust methodology, which would assess the genetic structure of the population and identify the existence of mixed stocks in the geographic area where the fishery is developed.

Key words: Penaeid, management unit, San Jorge Gulf.

1 Universidad Nacional de la Patagonia Austral, Unidad Académica Río Gallegos, Departamento de Ciencias Exactas y Naturales, Campus Universitario, Río Gallegos, Santa Cruz, Argentina.

2 Subsecretaría de Pesca, Ministerio de la Producción, Gobierno de la Provincia de Santa Cruz, Avellaneda 801, Río Gallegos, Santa Cruz, Argentina.

3 Fundación CEQUA CONICYT-Regional R07C1002. Av. 21 de Mayo 1690, Punta Arenas.

4 Laboratorio de Genética para la Conservación, Centro de Investigaciones Biológicas del Noroeste. Instituto Politécnico Nacional no. 195, La Paz, BCS, México.

* Este artículo forma parte de la tesis de P. De Carli para optar al título de Magister en Ciencias de la Universidad de Magallanes - Chile. pedro.decarli@gmail.com
\end{abstract}




\section{RESUMEN}

La pesquería de langostino argentino en Patagonia estuvo manejada desde su inicio como un único stock. Las investigaciones pesqueras realizadas consideraron que el concepto de unidad de stock y el seguimiento de cohorte, no es aplicable al manejo de la pesquería del langostino argentino. Los volúmenes anuales de captura de langostino presentaron importantes variaciones en sus números, que no han podido ser explicadas de modo efectivo. En este trabajo se analiza la factibilidad de reconocer diferentes subunidades de stock. La información biológica disponible, permite reconocer tres áreas núcleo de reproducción y crecimiento: litoral de Rawson $\left(43^{\circ} 30^{\prime} \mathrm{S}\right)$, norte del golfo San Jorge $\left(45^{\circ} \mathrm{S}\right)$ y sur del golfo San Jorge $\left(47^{\circ} \mathrm{S}\right)$. Los resultados disponibles de experiencias de marcado y recaptura, indican que una fracción de la población que nace y se desarrolla en la zona sur del golfo, permanece en esta zona y posiblemente efectúe allí su aporte reproductivo a la siguiente generación. Considerando la última década, en 2001 cuando las capturas anuales fueron máximas, se observó la concentración de la actividad de pesca en la zona litoral de Rawson, caracterizada por máximos rendimientos y tallas. Estas evidencias no resultan suficientes, y se requiere de otros métodos que permitan diferenciar las tres subunidades de stock propuestas. Se propone el análisis genético poblacional mediante marcadores moleculares como una metodología robusta, esto permitiría evaluar la estructura genética de la población, $e$ identificar la existencia de stocks mezclados en el área geográfica donde se desarrolla la pesquería.

\section{Palabras clave: Penaeido, unidad de manejo, golfo San Jorge}

\section{INTRODUCCIÓN}

El concepto de stock se ha desarrollado a partir de la actividad pesquera con la finalidad de evaluar la disponibilidad de recursos capturables, es considerado como un grupo de individuos de una especie susceptible de ser explotada en un área determinada (Saila \& Jones 1983, Milton \& Shaklee 1987) o como un grupo arbitrario de individuos que presentan historias de vida similares y en número suficiente para garantizar la reproducción (Hilborn \& Walters 1992); aunque existe una extensa revisión sobre el concepto de stock (Carvalho \& Hauser 1998, Hauser \& Ward 1998, Begg \& Waldman 1999, Ward 2000).

Desde el punto de vista de la conservación, la subestimación en la identificación de stock en el manejo pesquero que surge de considerar al recurso en explotación como un único stock, puede llevar a la pérdida de subpoblaciones y consecuentemente de biodiversidad intraespecífica (Viñas et al. 2011).

La pesquería de langostino argentino (Pleoticus muelleri, Bate 1888), concentra sus operaciones en la región patagónica atlántica comprendida entre las latitudes $42^{\circ}$ y $47^{\circ} \mathrm{S}$ y ocupa un lugar relevante en el sector pesquero de Argentina. El elevado valor comercial de este crustáceo en los mercados internacionales lo ha situado como uno de los principales productos de exportación pesquera de Argentina, representando una participación del 30\% en el valor de las exportaciones con un ingreso anual superior a los 300 millones de dólares estadounidenses ( $\mathrm{Di}$ rección de Economía Pesquera 2011¹).

La flota pesquera dedicada a la captura de langostino está constituida por aproximadamente 80 buques arrastreros congeladores, con esloras que varían entre 20 y 40 metros. Estas embarcaciones utilizan dos redes de arrastre de fondo de tipo marisqueras, una por banda, y realizan viajes de pesca de entre 20 y 60 días dependiendo de la capacidad de bodega y la disponibilidad del recurso.

A partir de la década de 1990 casi la totalidad (96\%) del desembarque declarado de esta especie proviene de la pesquería patagónica (Fischbach et al. 2006²), y mayoritariamente provienen de la flota congeladora tangonera (Góngora et al. 2009). La actividad de la flota se concentra en el golfo San Jorge y zona costera bajo la administración de tres jurisdicciones políticas diferentes: dos de orden pro-

1 Dirección de Economía Pesquera. 2011. Exportaciones pesqueras: comportamiento de los principales mercados. Secretaría de Pesca y Acuicultura. Ministerio de Agricultura, Ganadería y Pesca. Argentina. 20 pp.

2 Fischbach, C., J. De la Garza \& D. Bertuche 2006. La pesquería del langostino patagónico en el período 1991-2005. Informe Técnico INIDEP. $\mathrm{N}^{\circ}$ 03/06. $21 \mathrm{pp}$. 
vincial, correspondiente a Chubut y Santa Cruz, y la otra administrada por la autoridad de aplicación nacional.

En particular, la pesquería comercial de langostino en el golfo San Jorge comenzó a operar en la década de 1980, al mismo tiempo, el Instituto Nacional de Investigación y Desarrollo Pesquero (INIDEP) dio inicio a la investigación pesquera sobre el recurso, con tales investigaciones se propuso un modelo para explicar la dinámica del langostino argentino en aguas australes, según el cual la reproducción se concentra al norte del golfo San Jorge y el reclutamiento al sur del mismo golfo, implicando dos fenómenos dispersivos, uno de prerreclutas de norte a sur y otro de juveniles $y$ adultos desde el sur en dirección noreste (Fig. 1, Boschi 1989).

No obstante, estudios pesqueros posteriores pusieron de manifiesto que la reproducción tiene lugar a lo largo de todo el litoral patagónico entre

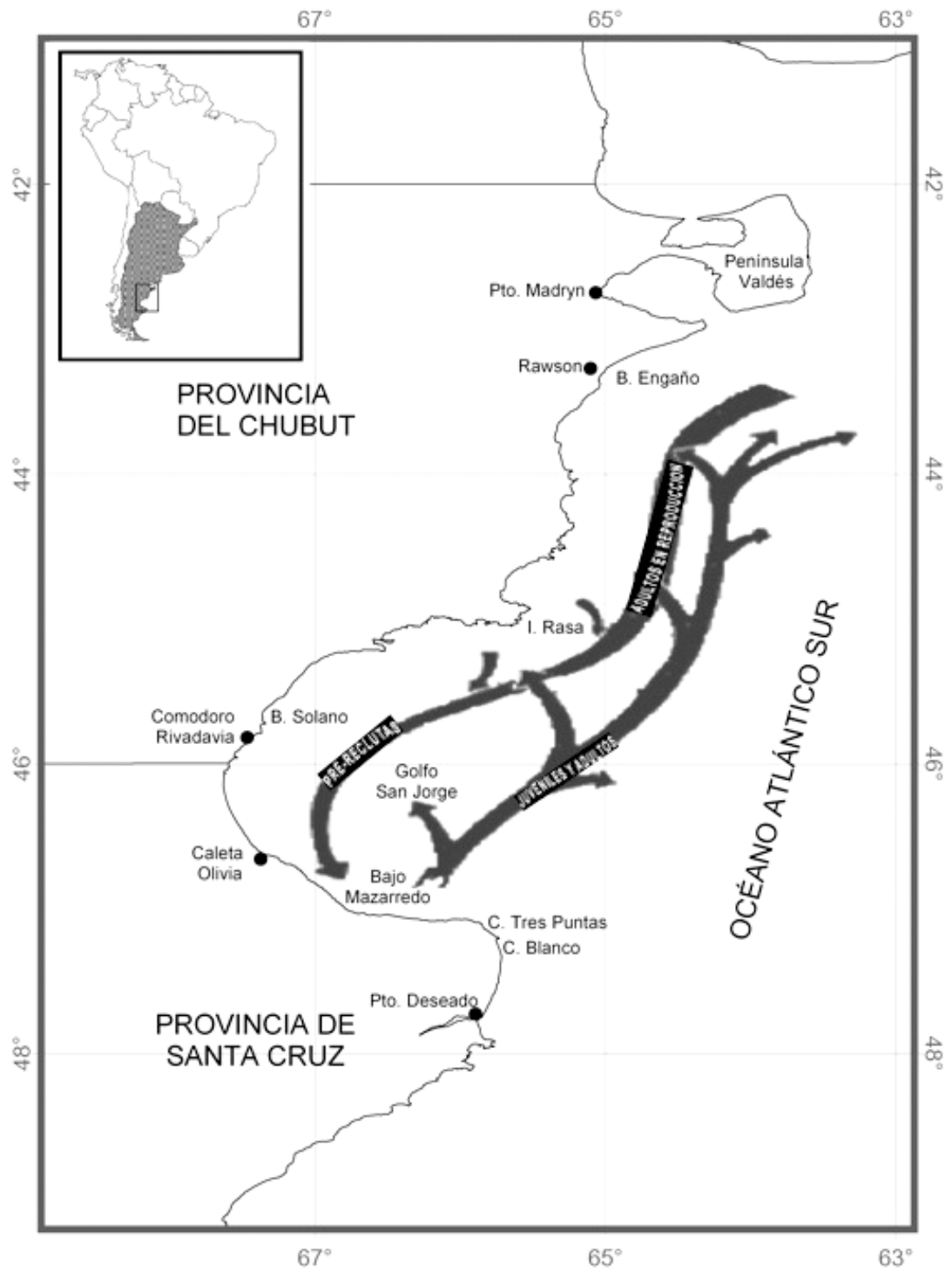

Fig. 1. Ciclo vital de langostino argentino del litoral patagónico, indicando las principales áeas de desove, desplazamiento y crecimiento (según Boschi 1989). 
las latitudes $42^{\circ}$ y $47^{\circ} \mathrm{S}$, concentrada en tres sectores litorales: sur de Rawson (43 $20^{\prime}$ al $44^{\circ} 20^{\prime} \mathrm{S}$, entre $64^{\circ} \mathrm{W}$ y la costa), norte del golfo San Jorge (desde isla Rasa hasta bahía Solano) y sur del golfo San Jorge (proximidad del bajo Mazarredo), en los que la estación reproductiva varía en cuanto a su inicio, duración e intensidad (Bertuche et al. 1996, Macchi et al. 1998, Fernández et al. 20014, 20025 Fernández 20056, Fernández \& Macchi 2007', Fernández et al. 2011).

Por su parte, Bertuche y Fischbach (1998) ${ }^{8}$ descartaron el uso de los conceptos de stock y cohorte para el análisis de la dinámica del langostino argentino, a pesar de ser ampliamente utilizados en el estudio de pesquerías de camarones tropicales. Sosteniendo que los procesos de reproducción, reclutamiento y crecimiento en $P$. muelleri ocurren de manera continua aunque variable en tiempo y espacio; y que por ello no es posible delimitar espacio temporalmente una cohorte o unidad de stock verdadera, para realizar su seguimiento durante períodos largos de tiempo.

El desarrollo de la pesquería patagónica de langostino conllevó la captura incidental de otras especies, al igual que ocurre con otras pesquerías de langostino y camarón en el mundo (Gillet 2008), siendo la merluza común (Merluccius hubbsi) la

3 Bertuche, D., C. Fischbach, M.I. Iorio \& M. Fernández 1996. La pesquería del langostino patagónico en 1996. Informe Técnico INIDEP N 94/96. 24 pp.

4 Fernández, M., G. Macchi \& J. De la Garza 2001. Estimación de fecundidad potencial y fecundidad relativa del langostino Pleoticus muelleri del Golfo San Jorge. Período analizado: Diciembre de 2000 a marzo de 2001. Informe Técnico INIDEPN ${ }^{\circ}$ 67/01. 21 pp.

5 Fernández, M., J. De la Garza \& G. Macchi 2002. Estimación de fecundidad potencial y fecundidad relativa del langostino Pleoticus muelleri del Golfo San Jorge y litoral de la Provincia de Chubut. Período analizado: Septiembre de 2001 a febrero de 2002. Informe Técnico INIDEP N³6/02. 33 pp.

6 Fernandez, M. 2005. Localización de las concentraciones reproductivas del langostino del litoral patagónico. Período analizado: diciembre de 2000 a enero de 2005. Informe Técnico INIDEP N ${ }^{\circ} 77 / 05.13$ pp.

7 Fernandez, M. \& G. Macchi 2007. Dinámica del proceso reproductivo del langostino del litoral patagónico. Resultados de la campaña de investigación OB-06/06 (noviembre de 2006). Informe Técnico INIDEP. 11 pp.

8 Bertuche D. \& C. Fischbach 1998. Respecto del manejo de la pesquería de langostino patagónico (Pleoticus muelleri) en el área sur del Golfo an Jorge en 1997/1998. Informe Técnico INIDEP $\mathrm{N}^{\circ}$ 72/98. $14 \mathrm{pp}$. especie más frecuente y abundante en estas capturas incidentales (Bertuche 1999, Pettovello 1999, Góngora et al. 2009, 2012, Góngora 2010 ${ }^{10}$ ).

En el año 1998 la autoridad de aplicación pesquera a nivel nacional decretó el estado de emergencia del recurso merluza común (Merluccius hubbsi), y estableció un área de veda permanente, para la protección de juveniles de merluza, en jurisdicción nacional frente al golfo San Jorge.

Anualmente, la pesquería de langostino inicia su actividad en el sur del golfo San Jorge entre los meses de marzo y abril. Hacia los meses de junio y julio, se habilita la captura de langostino en aguas exteriores del golfo San Jorge, en el área de veda de juveniles de merluza; y hacia el inicio de la primavera las capturas se concentran en el norte del golfo San Jorge y en el litoral de Chubut (cercano a la localidad de Rawson).

El objetivo del presente trabajo es analizar si la pesquería patagónica del langostino argentino está constituida por un solo stock, a partir de información biológica, ecológica y pesquera disponible.

\section{MATERIALES Y MÉTODOS}

La variación temporal de las capturas de langostino se analizó a partir de los volúmenes de desembarque anual declarado, obtenido de la estadística pesquera de la Subsecretaría de Pesca y Acuicultura de Argentina (Ministerio de Agricultura, Ganadería y Pesca 2011), y se identificó para esos años la ocurrencia de eventos "El Niño" (ENOS), a partir del Indice Oceánico del Niño (ONI) obtenido del National Weather Service (NOAA 2011) utilizado por el INIDEP para el análisis de las décadas de 1980 y 1990. Se graficaron los desembarques anuales de langostino para la serie 1981-2011, indicando los años de ocurrencia de ENOS.

Se realizó un análisis geoestadístico de las capturas usando el software DIVA-GIS v. 7.3. (Hijmans et al. 2004), el cual emplea el método de cuadriculas de vecindad (de 5 minutos de latitud por 5 minutos

9 Bertuche, D. 1999. Sobre la captura incidental de merluza en la pesquería de langostino patagónico. Informe Técnico INIDEP N $\mathrm{N}^{\circ}$ 76/99. $5 \mathrm{pp}$

10 Góngora, M.E. 2010. Dinámica y manejo de la captura incidental en la pesquería del langostino patagónico (Pleoticus muelleri). Tesis de doctorado. Universidad Nacional del Comahue. Centro Regional Universitario Bariloche. 208 pp. 
Tabla 1. Número de lances analizados por mes y año, del Programa de Observadores Pesqueros.

\begin{tabular}{|c|c|c|c|c|c|}
\hline Mes & 2001 & 2003 & 2005 & 2007 & Total \\
\hline E & 228 & 173 & & & 401 \\
\hline F & 700 & 272 & 2.467 & & 3.439 \\
\hline M & 359 & 1.503 & 1.579 & 1.816 & 5.257 \\
\hline A & 613 & 824 & 563 & 1.430 & 3.430 \\
\hline M & 523 & 782 & 660 & 1.526 & 3.491 \\
\hline J & 446 & 798 & 367 & 1.268 & 2.879 \\
\hline J & 707 & 1.092 & 20 & 864 & 2.683 \\
\hline A & 602 & 1.243 & & 1.108 & 2.953 \\
\hline S & 250 & 1.283 & & 783 & 2.316 \\
\hline $\mathrm{O}$ & 514 & 720 & 409 & 274 & 1.917 \\
\hline $\mathrm{N}$ & 94 & 1.262 & 296 & & 1.652 \\
\hline $\mathrm{D}$ & 116 & 423 & 826 & & 1.365 \\
\hline Total & 5.152 & 10.375 & 7.187 & 9.069 & 31.783 \\
\hline
\end{tabular}

de longitud). Las capturas de langostino y merluza fueron obtenidas mediante estimación volumétrica visual por observadores a bordo de los Programas de Observadores Pesqueros (POP). Las capturas estimadas por lance se transformaron en captura por unidad de esfuerzo (CPUE) de langostino, al relacionar la biomasa (en kilogramos) y el tiempo de arrastre (en horas), y se calculó el porcentaje de merluza común en relación a la captura total por lance.

Los registros del POP consistieron en datos de: captura de langostino y captura incidental de merluza común, obtenidos a bordo de buques pesqueros tangoneros en la zona comprendida entre las latitudes $43^{\circ}$ y $47^{\circ} \mathrm{S}$, en los años 2001,2003 , 2005 y 2007, en que se dispone de información con grado similar de cobertura (Tabla 1).

También se realizó una revisión de información de biología pesquera del langostino argentino, a partir de informes técnicos producidos por el INIDEP de Argentina.

\section{RESULTADOS}

Las capturas de langostino a partir de los desembarques totales declarados para el período 1994-2010 presentaron un valor medio de 33.800 toneladas anuales, con un desvío de 22.200 toneladas, con registros máximos en los años 2001 y 2010 mayores a las 70.000 toneladas, y mínimos en los años 1995 y 2005 con desembarques cercanos a las 7.000 toneladas (Fig. 2).

Los desembarques anuales registraron hasta el año 2000 valores menores a las 30.000 toneladas
(Fig. 2). Analizando todo su historial se observó que presentan una distribución multimodal, con modas en los años 1984, 1988, 1992, 2001 y 2010, y mínimos en los años 1987, 1995 y 2005 . Además, en los años 1982, 1987, 1991, 1997 y 2009 los ENOS antecedieron en doce meses al incremento en los volúmenes de captura, pero no fue así para los años 2002, 2004 y 2006, en donde incluso precedieron a registros mínimos históricos en las capturas anuales.

El análisis geoestadístico de los datos de captura por unidad de esfuerzo en el año 2001, en que se registraron las máximas capturas anuales declaradas en la historia de la pesquería, muestran que en la zona frente a Rawson (entre los $43^{\circ}$ y $45^{\circ}$ S) se presentaron los mayores valores de captura por unidad de esfuerzo para langostino (kilogramos hora-1), y los menores valores de porcentaje de merluza en relación a la captura total por lance (Fig. 3.a. y 4.a.). Este patrón no se observó en el año 2007 (Fig. 3.d. y 4.d.). Para los años 2003 y 2005 esta zona estuvo cerrada a la pesca de langostino debido a la veda de juveniles de merluza en las aguas exteriores de jurisdicción nacional (Fig. 3.b., 3.c., 4.b. y 4.c.).

Sin embargo, para los años 2003 y 2007 (Fig. 3.b. y 3.d.) los análisis indican que el patrón de pesca fue disperso a lo largo del golfo San Jorge con concentraciones importantes de las capturas entre los meridianos $65^{\circ} 30^{\prime}$ y $66^{\circ} 10^{\prime} \mathrm{W}$, en el sur del golfo, y entre los $64^{\circ}$ y $65^{\circ} 30^{\prime} \mathrm{W}$ aproximadamente en el sector norte, y ausencia de desarrollo de la pesquería en la zona de Rawson. 


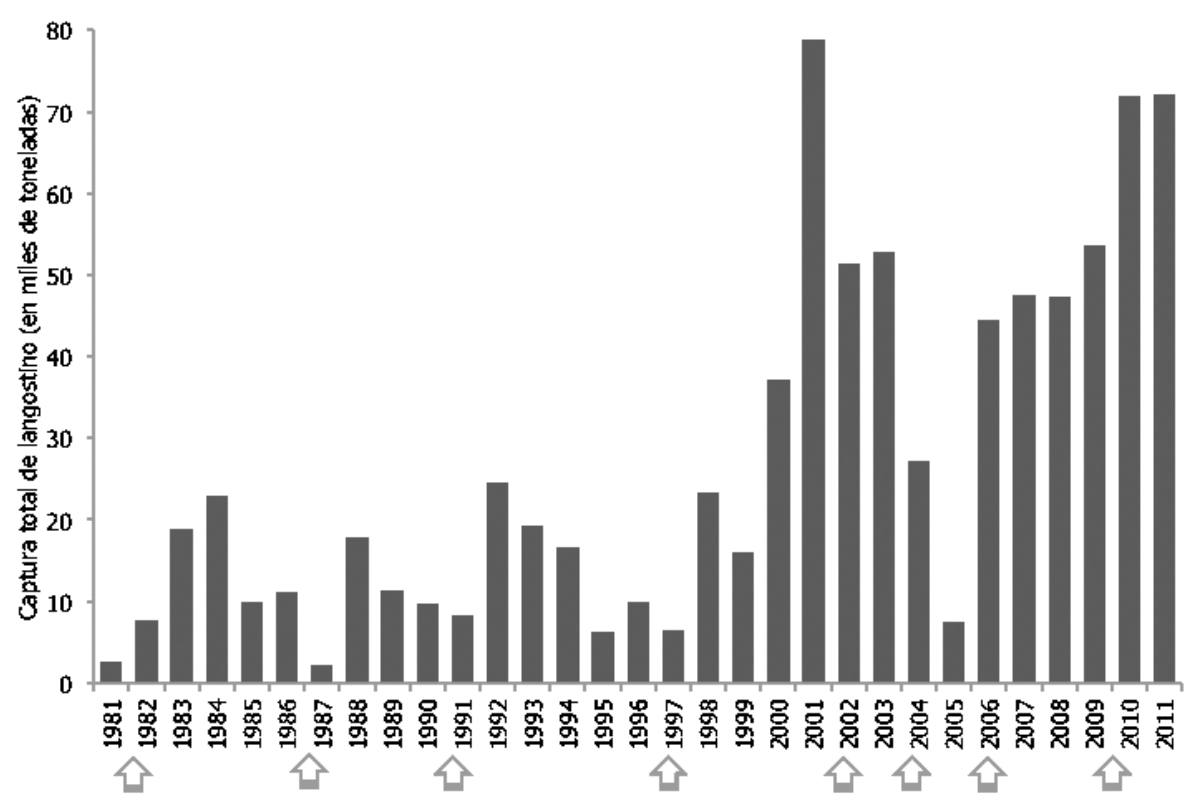

Fig. 2. Desembarques totales declarados de langostino entre 1981 y 2011 (se indica con una flecha la ocurrencia de eventos "El Niño" - ENSO).

En particular para el año 2007, se presentó una concentración de capturas superiores a los 5.000 $\mathrm{kg} \mathrm{h}^{-1}$ entre los meridianos $64^{\circ}$ y $65^{\circ} \mathrm{W}$ en la latitud $46^{\circ} \mathrm{S}$ (Fig. 3.d.). En tanto esos rendimientos fueron alcanzados en el año 2001 entre las latitudes $43^{\circ}$ $30^{\prime}$ y $44^{\circ} 30^{\prime} \mathrm{S}$ y entre los meridianos $63^{\circ}$ y $65^{\circ}$ W (Fig. 3.a.).

En el año 2005, donde el año anterior se registraron las anomalías de El Niño según índice ONI (National Oceanic and Atmospheric Administration 2011), las capturas alcanzaron el mínimo histórico anual (Fig. 2). Asimismo se observó la mayor concentración de la captura por unidad de esfuerzo (CPUE) en jurisdicción de Santa Cruz entre las latitudes $46^{\circ} 30^{\prime}$ y $47^{\circ} \mathrm{S}$, en proximidad del meridiano $66^{\circ} \mathrm{W}$. En las zonas de jurisdicción nacional y de la provincia del Chubut las capturas se presentaron dispersas y con predominancia de rendimientos menores a los $500 \mathrm{~kg} \mathrm{~h}^{-1}$ (Fig. 3.c.).

\section{DISCUSIÓN Y CONCLUSIONES}

En la búsqueda por desarrollar un modelo estadístico que permita explicar las variaciones en la captura media por unidad de esfuerzo en relación a variables ambientales del contexto local y global,
Bertuche et al. (2000) ${ }^{11}$ estimaron una regresión múltiple significativa con tres variables importantes: 1) el esfuerzo total aplicado; 2) la anomalía de temperatura de superficie del mar en el golfo San Jorge; y 3) la anomalía de la temperatura de superficie del mar en la costa peruana doce meses antes de la captura, que describe al fenómeno de "El Niño - Oscilación del Sur" (ENOS). Si bien se había observado para las décadas de 1980 y 1990 , analizadas por Bertuche y colaboradores, que los años de ENOS anteceden a años de incremento en las capturas de langostino; al incorporar al análisis la última década (2000-2010) no se verifica este patrón con posterioridad a los ENOS de los años 2002 y 2004 sino por el contrario, ya que este último caso antecede a un año de captura mínima histórica para la pesquería (Fig. 2).

El langostino argentino se caracteriza por un ciclo de vida relativamente corto y una tasa de crecimiento alta y variable, un gran potencial reproductivo, $y$ habitualmente con un reemplazo

11 Bertuche, D., C. Fischbach \& J. De la Garza 2000. La fluctuación de la abundancia de langostino en el Golfo San Jorge. Un análisis preliminar de su relación con el manejo pesquero y los cambios del contexto climático global. Informe Técnico INIDEP № 45/00. 15 pp. 

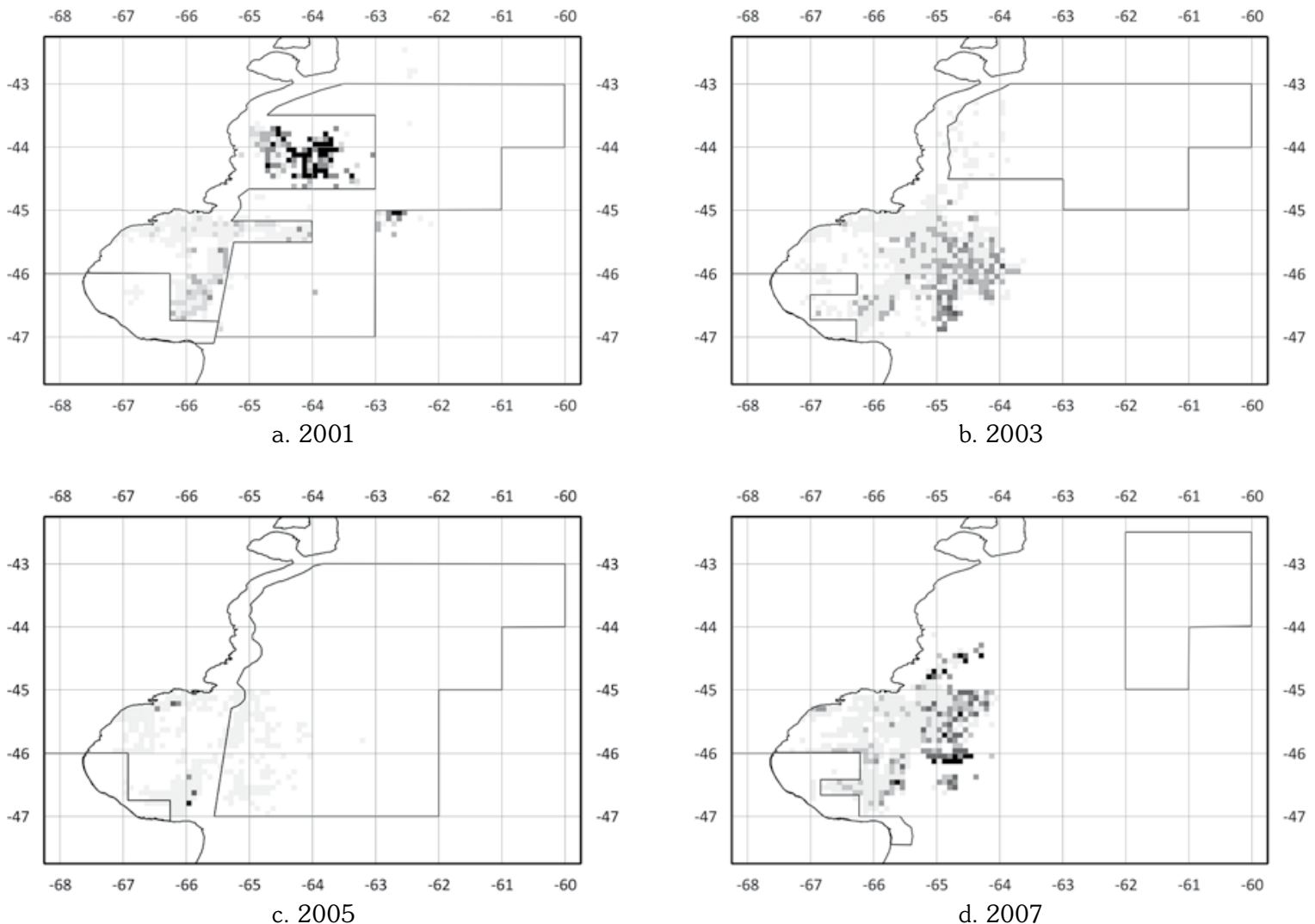

Fig. 3. Distribución geográfica de la captura por unidad de esfuerzo (CPUE) en valores modales de kg de langostino / hora de arrastre del lance de pesca, por cuadro de $\left(5^{\prime} \times 5^{\prime}\right)$, para los distintos años analizados (cada inciso corresponde al año de captura que se indica, $\square$ zonas de veda, $\square 1-500, \square 501-1.000, \square 1.001-2.500, \square 2.501-5.000, \square 5.000-60.000$ ).

casi total de la biomasa disponible entre dos años de pesca sucesivos. Según Bertuche $(1998)^{12}$ estas características, junto con la fluctuación del nivel de reclutamiento anual a la pesca comercial, generan la variabilidad observada en la abundancia de la biomasa disponible para la flota langostinera, poniendo al recurso en permanente riesgo de sobrepesca en la fase de crecimiento y de sobrepesca en la fase de reclutamiento (Bertuche \& Fischbach, 1998 ${ }^{8}$, Fischbach \& Bertuche, 199913, De la Garza, 200614).

12 Bertuche, D. 1998. Campaña OB-01/98. Evaluación de langostino. Informe de campaña INIDEP. $11 \mathrm{pp}$.

13 Fischbach, C. \& D. Bertuche 1999. Un análisis de la dinámica de la cosecha de langostino patagónico en la temporada de 1998. Informe Técnico INIDEP N³8/99. 15 pp.

14 De la Garza, J. 2006. Estimación de los parámetros de crecimiento del langostino patagónico utilizando frecuencias de tallas para las temporadas 2004 a 2006. Informe Técnico INIDEP $\mathrm{N}^{\circ}$ 78/06. $13 \mathrm{pp}$.
En el año 2001, en que la captura anual de langostino argentino alcanzó un máximo histórico de 78.800 toneladas, la disposición espacial de la CPUE manifestó un patrón de concentración en la zona costera próxima a la localidad de Rawson $\left(44^{\circ}\right.$ S). Allí se registraron rendimientos en captura por unidad de esfuerzo mayores a los valores medios para ese año (Fig. 3.a.). Para esta misma área se reportaron las mayores tallas en longitud de cefalotórax para la pesquería (Ruiz \& Mendía, 2008). Estas observaciones permiten plantear la hipótesis de que, el incremento en las capturas anuales en años de mayor abundancia se debe al aporte de individuos provenientes de una zona exógena a los fondos de pesca, con individuos que presentan un mayor tamaño corporal.

La administración pesquera de Argentina en el año 1998 declaró al recurso merluza común (Merluccius hubbsi) en estado de emergencia al 

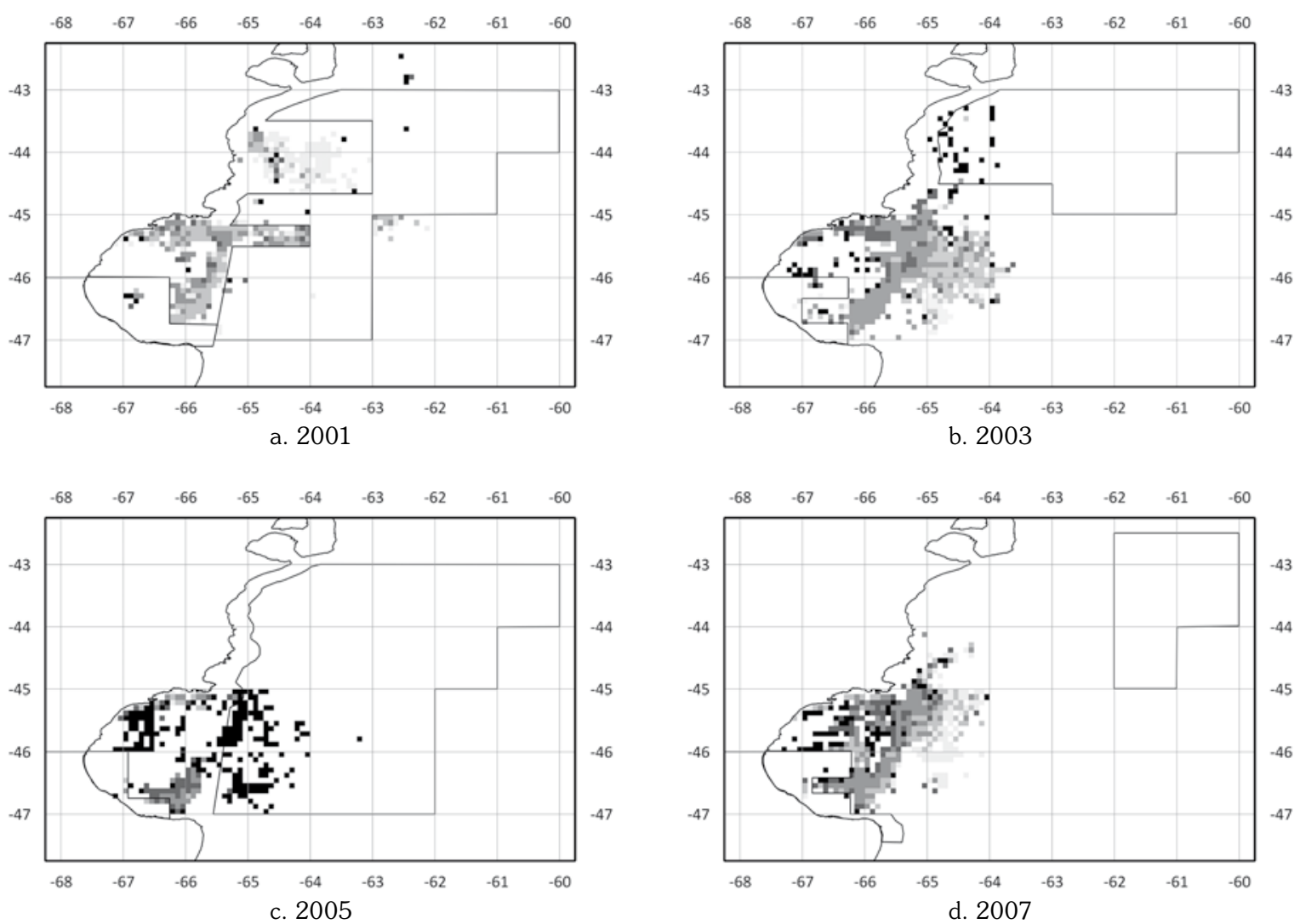

Fig. 4. Distribución geográfica del porcentaje de merluza como captura incidental en relación a la captura total por lance, en valores medios por cuadro de $\left(5^{\prime} \times 5^{\prime}\right)$, para los distintos años analizados (cada inciso corresponde al año de captura que se indica, $\square$ zonas de veda, $\square, 00-0,20, \square 0,21-0,40, \square 0,41-0,60, \square 0,61-0,80, \square 0,81-1,00)$.

detectar signos de sobreexplotación, y estableció a partir del mismo año un área de veda permanente entre las latitudes $43^{\circ}$ y $47^{\circ} \mathrm{S}$. Anualmente, en determinadas épocas se habilitaron áreas para la pesca de langostino argentino dentro de la zona de veda. Tal disposición restringió el área de la actividad de pesca de langostino argentino, en algunos años limitó su actividad a aguas interiores del golfo San Jorge (Fig. 3.a. y 3.c.), y en otros años en aguas interiores y exteriores adyacentes al golfo San Jorge (Fig. 3.b.). Estas restricciones afectaron la captura del langostino argentino al limitar la libre operatividad de la flota, produciendo como consecuencia la disposición espacial de la CPUE observada en este estudio para los años 2003 y 2005.

Mientras el modelo desarrollado por Boschi (1989) proponía una única unidad poblacional, las evidencias biológicas, ecológicas y pesqueras posteriores
(Iorio et al. 200015, Fernández et al. 20014, 2002 Fernández, 2005 , Fernández \& Macchi, 20077) permiten postular la hipótesis de la existencia de tres subunidades desde el punto de vista ecológico: una al norte del golfo San Jorge ( $\left.45^{\circ} \mathrm{S}\right)$, otra al sur del golfo San Jorge $\left(47^{\circ} \mathrm{S}\right)$ y la tercera en aguas exteriores al golfo, en la zona de Rawson ( $\left.43^{\circ} \mathrm{S}\right)$.

Por otra parte, se conoce que el período de desove en el sur del golfo San Jorge se desarrolla entre diciembre y abril, mientras que en el norte del golfo se inicia en octubre y se extiende hasta marzo, y en el litoral sur de Rawson entre octubre y diciembre. Este comportamiento reproductivo se ha relacionado con el gradiente latitudinal de temperatura y la evolución

15 Iorio, M.I., G.J. Macchi \& D. Hernández 2000. Estimación de la talla de primera madurez y fecundidad del langostino patagónico. Caracterización del desarrollo ovárico y el estadío de impregnación. Informe Técnico INIDEP № 61/00. 15 pp. 
de los procesos ambientales asociados (Fernández et al. 20014). La segregación espacio temporal del comportamiento reproductivo sustenta la hipótesis propuesta en relación a distinguir tres subunidades en la población de langostino argentino.

Además, la distribución espacial del desarrollo larval y el crecimiento incorpora nuevas evidencias que contribuyen a reforzar la hipótesis planteada. Así por ejemplo, el área de distribución de los primeros estadios de desarrollo se amplía a medida que la larva comienza a asociarse al fondo y se convierte en juvenil, este desarrollo se da en tres lugares de crecimiento: el primero se localiza en el área de Isla Escondida (43 $30^{\prime} \mathrm{S}$ y $64^{\circ} 20^{\prime} \mathrm{W}$ a la costa), la segunda en la parte norte del golfo San Jorge $\left(45^{\circ}\right.$ $20^{\prime}$ y $45^{\circ} 40^{\prime} \mathrm{S}$; y $64^{\circ} 55^{\prime}$ y $66^{\circ} 30^{\prime} \mathrm{W}$ ), y la tercera en el sur en el Bajo de Mazarredo $\left(46^{\circ} 26^{\prime}\right.$ y $46^{\circ}$ $56^{\prime}$ S; y $66^{\circ} 20^{\prime}$ y $67^{\circ} 09^{\prime}$ W - Fig. 1), donde estos lugares se corresponden a los tres stock propuestos.

Asimismo, en la zona denominada como de "la pared", en el sudeste del golfo San Jorge $\left(46^{\circ} 45^{\prime}\right.$ S, $65^{\circ} 55^{\prime} \mathrm{W}$ y $46^{\circ} 55^{\prime} \mathrm{S}, 65^{\circ} 55^{\prime} \mathrm{W}$ ), se han encontrado concentraciones de huevos de langostino, que permitirían inferir la presencia de concentraciones de langostino en estado reproductivo (De la Garza 200716, De la Garza et al. 2008 ${ }^{17}$, Moriondo 200618, Moriondo \& Ravalli $2007^{19}$ ), en esta zona los fondos de arrastre están limitados a áreas de pequeña extensión que resultan poco accesibles a la flota pesquera y a las campañas de investigación. Por otra parte, el área de muestreo de las campañas del INIDEP se extiende entre las latitudes $42^{\circ}$ y $47^{\circ} \mathrm{S}$ y la longitud $62^{\circ} \mathrm{W}$ y la costa; mientras que se ha reportado presencia de langostino desde los $23^{\circ} \mathrm{S}$ (Boschi, 1997) hasta los $50^{\circ} \mathrm{S}$ (Bertuche, $1998^{12}$ ), por lo que la extensión del

16 De la Garza J. 2007. Informe de la Campaña OB-02/07. Proyecto Langostino. 25 pp.

17 De la Garza J, D Cuchi Colleoni, A Izzo, J Boccanfuso, C Bertelo \& G Cano. 2008. Campaña de relevamiento de langostino patagónico (Pleoticus muelleri) en el golfo San Jorge a bordo de un buque comercial. Proyecto Langostino. $18 \mathrm{pp}$.

18 Moriondo P. 2006. Distribución y abundancia de huevos y larvas de langostino patagónico (Pleoticus muelleri) y su relación con los parámetros ambientales. Resultados de las campañas OB 10/05 y OB 01/06. Informe Técnico INIDEP $\mathrm{N}^{\circ}$ 63/06. 14 pp.

19 Moriondo P. \& C. Ravalli 2007. Distribución y abundancia de huevos y larvas de langostino patagónico (Pleoticus muelleri). Resultado de las campañas OB 06/06 y OB 02/07. Informe Técnico INIDEP N 45/07. 10 pp. área de repoducción en la zona sur del golfo podría ser más extensa de lo que se había descrito.

A partir del año 2001, el INIDEP inició un programa de marcado, liberación y recaptura de langostino argentino en aguas del golfo San Jorge y áreas adyacentes, con el objetivo de establecer los principales patrones de migración. A partir de las recapturas se pudo identificar dos rutas migratorias que se desarrollan desde el área de Mazarredo (ubicada entre la latitud $46^{\circ} 30^{\prime} \mathrm{S}$ y la costa y las longitudes $66^{\circ} 10^{\prime}$ y $67^{\circ} \mathrm{W}$ ), ambas con dirección N-NE: una de larga distancia, con individuos recapturados al norte de la latitud $44^{\circ} \mathrm{S}$, y otra de corta distancia, con individuos recapturados en la zona de la pared al sur de la latitud $46^{\circ} \mathrm{S}$ (Roux et al. $2012^{20}$ ). Esta dinámica de migración indica que una fracción de la población que se desarrolla y crece en la zona sur del golfo, permanece en esta zona y posiblemente efectúe ahí su aporte reproductivo a la siguiente generación, por lo que este grupo poblacional podría constituir uno de los stock previamente descritos.

Como se mencionó, Bertuche y Fischbach $(1998)^{8}$ consideraron que no es posible aplicar el concepto de unidad de stock y el seguimiento de cohorte para el manejo de la pesquería del langostino argentino, debido a que los procesos de reproducción, reclutamiento y crecimiento ocurren de manera continua y variable en tiempo y espacio. Sin embargo, las evidencias presentadas en este estudio parecen contradecir esta idea.

Debido a las dificultades puestas de manifiesto por Bertuche y Fischbach $(1998)^{8}$ para aplicar el concepto clásico de stock y seguimiento de cohorte, se requiere de otros métodos que permitan su reconocimiento. El análisis genético poblacional usando marcadores moleculares representa una metodología robusta para tales fines, esta aproximación permitiría evaluar si existe estructuración genética de la población e identificar la existencia de stocks mezclados en el área geográfica donde se desarrolla la pesquería.

\section{AGRADECIMIENTOS}

Los autores quieren expresar su agradecimiento al Programa de Observadores a Bordo de las provincias de Chubut, Santa Cruz y el INIDEP.

20 Roux A., J. De la Garza, R. Piñero \& D. Bertuche. 2012. La ruta migratoria del langostino patagónico. Informe Técnico INIDEP $\mathrm{N}^{\circ}$ 07/12. 24 pp. 
El presente trabajo forma parte del proyecto de investigación de Tesis de P. De Carli para optar al Título de Magister en Ciencias con mención en Manejo y Conservación de Recursos en ambientes Subantárticos de la Universidad de Magallanes (Punta Arenas - Chile), recibió apoyo de la Subsecretaría de Pesca (Ministerio de la Producción - Gobierno de la provincia de Santa Cruz - Argentina), del proyecto SECYT-UNPA 29A/231 de la Universidad Nacional de la Patagonia Austral (Río Gallegos - Argentina), del Centro Regional de Estudios del Cuaternario (Fundación CEQUA, Punta Arenas - Chile). FJGdL agradece al CIBNOR por su apoyo en el año sabático.

\section{LITERATURA CITADA}

Begg, G.A. \& J.R. Waldman 1999. An holistic approach to fish stock identification. Fisheries Research, 43: 35-44.

Boschi, E.E. 1989. Biología pesquera del langostino del litoral patagónico de Argentina (Pleoticus muelleri). Contribuciones INIDEP 646: 1-71.

Boschi, E.E. 1997. Las pesquerías de crustáceos decápodos en el litoral de la República Argentina. Investigación Marina. Valparaiso 25: $19-40$.

Carvalho, G.R. \& L. Hauser 1998. Advances in the molecular analysis of fish population structure. Italian Journal of Zoology 65 Suppl.: 21-33.

Fernandez, M., D. Hernandez \& A. Roux 2011. Analysis of the relationship between relative abundance of mature, impregnated females of Pleoticus muelleri (Bate, 1888) (Crustacea, Decapoda) and environmental variables through statistical models. Latin American Journal of Aquatic Research, 39 (1): 1-15.

Gillet, R. 2008. Global study of shrimp fisheries. Food and Agriculture Organization of the United Nations. Fisheries Technical Paper $N^{\circ}$ 475. Roma. $331 \mathrm{pp}$.

Góngora, M.E., N.D. Bovcon \& P.D. Cochia 2009. Ictiofauna capturada incidentalmente en la pesquería de langostino patagónico Pleoticus muelleri, Bate 1888. Revista de Biología Marina y Oceanografía, 44 (3): 583-593.

Góngora, M.E., D. Gonzalez-Zevallos, A. Pettovello \& L. Mendía 2012. Caracterización de las principales pesquerías del golfo San Jorge Patagonia, Argentina. Latin American Journal of Aquatic Research, 40 (1): 1-11.
Hauser, L. \& R.D. Ward 1998. Population identification in pelagic fish: the limits of molecular markers. In: Advances in Molecular Ecology (ed. G.R. Carvalho). IOS Press, Amsterdam, pp. 191-224.

Hijmans, R.J., L. Guarino, C. Bussink, P. Mathur, M. Cruz, I. Barrentes \& E. Rojas 2004. DIVA-GIS. Vsn. 5.0. A geographic information system for the analysis of species distribution data. Manual available at http://www.diva-gis.org.

Hilborn, R. \& C.J. Walters 1992. Quantitative Fisheries Stock Assessment. Choice, Dynamics and Uncertainty. Chapman \& Hall, New York, 570 pp.

Macchi, G., M.I. Iorio \& A. Aubone 1998. Estimación de la fecundidad del langostino argentino Pleoticus muelleri Bate, 1888 de Patagonia (sur de Argentina). 14 (1 y 2): 19-29.

Milton, D.A. \& J.B. Shaklee 1987. Biochemical genetics and population structure of blue grenadier, Macruronus novaezelandiae (Hector) (Pisces: Merluccidae), from Australian waters. Australian Journal of Marine and Freshwater Research, 38: 727-747.

Ministerio de Agricultura, Ganadería y Pesca. 2011. Desembarques de pesca marítima. En: http://www.minagri.gob.ar/site/ pesca/ pesca_maritima/02-desembarques/index.php.

Pettovello, A. 1999. Bycatch in the Patagonian red shrimp (Pleoticus muelleri) fishery. Marine and Freshwater Research 50: 123-127.

Ruiz, A.E. \& L.F. Mendia 2008. Observaciones morfométricas y reproductivas en el langostino Pleoticus muelleri Bate, 1888 procedente de embarcaciones comerciales del puerto Rawson, Argentina. Revista de Biología Marina y Oceanografía 43 (3): 675-680.

Saila, S. \& C. Jones 1983. Fishery science and the stock concept. Final Report P.O. NA83-BA-0078 (MS) US National Marine Fisheries Service, Northeast Fisheries Center, Woods Hole, MA 02543.

Viñas, J., A. Gordoa, R. Fernández Cebrián, C. Pla, U. Vahdet \& R.M. Araguas 2011. Facts and uncertainties about the genetic population structure of Atlantic bluefin tuna (Thunnus thynnus) in the Mediterranean. Implications for fishery management. Reviews in Fish Biology and Fisheries, 21 (3): 527-541.

Ward, R.D. 2000. Genetics in fisheries management. Hydrobiologia, 420: 191-201. 